\title{
Adolescent idiopathic scoliosis and back pain
}

Federico Balagué ${ }^{1,2,3^{*}}$ and Ferran Pellisé $e^{4,5^{*}}$

\begin{abstract}
This broad narrative review addresses the relationship between adolescent idiopathic scoliosis (AIS) and back pain. AIS can be responsible for low back pain, particularly major cases. However, a linear relationship between back pain and the magnitude of the deformity cannot be expected for any individual patient. A large number of juvenile patients can remain pain-free. The long-term prognosis is rather benign for many cases and thus a tailored approach to the individual patient seems mandatory. The level of evidence available does not allow stringent recommendations for any of the disorders included in this review.
\end{abstract}

Keywords: Adolescent idiopathic scoliosis, Back pain, Conservative management, Surgical treatment, Natural history

\section{Background}

Scoliosis is a frequent pathology in adolescents. Young patients and their families frequently blame (minor) deformities, considered to be the cause of back pain. In our opinion, the relationship between the two is not so clear based on scientific evidence. For this reason, we here review this topic in depth.

A high prevalence of low back pain (LBP) among children and adolescents has been identified in the last few decades. A recent systematic review and meta-analysis [1] has reported a figure of $39.9 \%$ (95 \% CI ranging from 34.2 to $45.9 \%$ ) for lifetime prevalence. In terms of aetiologies, a retrospective study of almost 2000 patients less than 21 years-old referred for a spine evaluation reported that when a pathology is identified, the most frequent diagnosis was scoliosis (1439/1953), followed by Scheuermann's kyphosis (163/1953) and spondylolisthesis (154/1953) [2]. Other series of cases have also shown similar findings $[3,4]$. However, the role of spinal deformities in LBP in the general population is not clear. For this reason, we here review the recent literature on the associations between pain and the most common adolescent spinal deformity, which is adolescent idiopathic scoliosis.

Trying to establish a causal relationship between deformities and back pain is not an easy task. Since Sir Austin

\footnotetext{
* Correspondence: Federico.Balague@h-fr.ch; 24361fpu@comb.cat

${ }^{1}$ Department of Rheumatology, HFR Fribourg-Hôpital Cantonal, 1708 Fribourg, Switzerland

${ }^{4}$ Spine Unit, Hospital Vall Hebron, 08035 Barcelona, Spain

Full list of author information is available at the end of the article
}

Bradford Hill [5] published his important paper in 1965, the difficulties and limitations in drawing conclusions on the causal relationship between two variables have been the focus of several publications [6-9].

Our aim is to review the literature to evaluate the association in adolescents between back pain and idiopathic scoliosis, reported in studies done in different settings and/or with different perspectives.

\section{Methodology}

This paper is the product of the collaboration between 2 clinicians: an orthopaedic surgeon highly focused on deformities (FP) and a rheumatologist with a special interest in juvenile spinal disorders (FB). We have tried to find clinically relevant information that might answer some of the questions the practitioners have. Therefore this article does not aim to be a systematic review as we have not applied strict methodology as recommended, for example, by the Cochrane collaboration, for such reviews. We began with a bibliography search limited to MEDLINE and expanded this body of literature with a search of the publications cited in the selected articles. The search was done using several key words for back pain (backache OR back pain OR low back pain OR lumbar pain OR vertebral pain OR spinal pain) as well as for the age category of interest (adolescent OR teenager OR juvenile OR paediatric OR infant OR children). We then limited the search to metaanalyses and expanded it to include "systematic reviews", and "cohort or longitudinal studies", if the information 
gathered was too limited with the most stringent criteria. Our focus was on back pain and consequently the articles not including data on symptoms were excluded except for the Epidemiology section.

\section{Adolescent idiopathic scoliosis (AIS) and back pain}

According to the glossary of the Scoliosis Research Society (SRS) (http://www.srs.org/professionals/online-educationand-resources/glossary/revised-glossary-of-terms), idiopathic scoliosis can be defined radiographically as a lateral curvature of the spine greater than or equal to $10^{\circ} \mathrm{Cobb}$ with rotation, of unknown aetiology.

The possible relationship between scoliosis and back pain can be approached from different angles. Some examples may be:

a) comparing the epidemiological data of the two diagnostic entities;

b) examining the prevalence of back pain in adolescents with scoliosis or the prevalence of scoliosis among back pain sufferers;

c) examining the prevalence of back pain over time in scoliosis left untreated; or

d) examining the impact of treatment of the deformity on back pain.

\section{Epidemiology}

\section{Prevalence of scoliosis}

Adolescent idiopathic scoliosis is considered a quite common disorder, with a pooled referral rate for radiography of $5.0 \%$, according to a meta-analysis of 36 studies looking at the effectiveness of school scoliosis screening [10]. According to the definition used, the overall prevalence of AIS has been reported to range between 0.47 and $5.2 \%$ in a recent review [11]. There are cases of scoliosis secondary to other pathologies, but those idiopathic (i.e. AIS) are by far the most frequent. In the series published by Rogala et al. [12], all but 9 of 1231 cases of structural scoliosis were idiopathic. We have limited this section of the review to adolescents or young adults with AIS.

There are other studies that have prospectively evaluated the epidemiology of scoliosis and its natural history in large samples of adolescents, but the most frequently referenced do not include any information on symptoms [11-15]. Even the recent meta-analysis previously mentioned did not report data on back pain [10].

Scoliosis can be associated with other deviations from the normal spinal morphology. For example, in the series reported by Deacon et al. [16], 35 out of 50 adolescents were diagnosed with both Scheuermann's disease and scoliotic curves. Overall, 43 curves were identified in these patients, which were divided in 2 types: 13 were apical at the same level as the kyphosis, and 30 occurred in regions of compensatory lordosis. Finally, in the small series reported by Blumenthal et al. [17], 4 out of 13 cases of lumbar Scheuermann's also had scoliosis but none required treatment.

\section{Pain associated with scoliosis}

Regarding associated symptoms, a German study evaluated the data of more than 640,000 youths included in an insurance database. ICD diagnosis codes M40-43 would be relevant for the purpose of our review (M40: kyphosis and lordosis; M41: scoliosis; M42: spinal osteochondrosis; and M43: other deforming dorsopathies). For scoliosis, data from 2002 showed the following means in \% (girls/ boys) for prevalence at $0-14$ years of age and at $15-24$ years: M41: $2.31(2.51 / 2.12)$ and 3.44 (3.80/3.07) [18]. Ramirez et al. [19] reported on more than 2400 subjects with AIS. Of these, $23 \%$ reported back pain at the time of diagnosis. An additional $9 \%$, initially free of pain and managed with observation alone, developed pain during follow-up. A study from Japan including more than 30,000 adolescents concluded that the subgroup with scoliosis had an approximately 3 to 5 fold increased point and lifetime prevalence of backache in the upper and middle right part of the back [20]. In a prospective multicentre study, Lonner et al. [21] compared three groups of adolescents including 894 with AIS and 31 healthy controls. The pain scores on the specific subdomain of the SRS-22 questionnaire were 4.15 in the scoliosis group and 4.24 among the controls, which is not a significant difference.

A retrospective chart review of a random sample of 310 individuals (10-17 years) selected from all cases of AIS referred to a Canadian university hospital has been published recently [22]. The authors concluded that the prevalence of back pain was "moderately high" but the reported data on pain do not seem very homogeneous (gathered by the attending physicians, or reported by medical references, or by letters from the parents, narrative or through a pain score, with or without a specific topography of pain recorded, etc.). Of note, severe pain was documented in only $1 \%$ of the charts [22].

A prospective multicentre study including 744 patients (621 females) surgically treated for AIS addressed the differences between genders in functional outcome. Before surgery, males were aged an average of 15.2 years and females 14.0 years $(p<0.001)$ with no significant differences in maximum Cobb angles (F: $53.3^{\circ}$ and M: 55.9 $)$ or Risser grades (F: 3.2 and M: 3.5) [23]. At baseline, the scores on the pain domain of the SRS-30 were 4.1 among girls and 4.3 in boys, below the statistical significance threshold [23].

A recent meta-analysis has been published comparing selective versus non-selective thoracic fusion in Lenke 1C curves [24]. Preoperative data on pain using the SRS-22 has shown slightly different scores between the two 
groups that is $4.13(0.77)$ in the selective fusion group versus $3.92(0.79)$ in those undergoing non-selective procedures. The difference is statistically significant $(p=$ 0.038 ), but is at the limit of the minimum clinically important difference (MCID), reported to be 0.20 [21, 25, 26]. As any other tool, the questionnaires used to gather information on patients with scoliosis have some limitations. An American study evaluated SRS-22 performance in 450 healthy adolescents (mean age: 16 years; range: 9.3 to 21.8 years). Concerning specifically pain, the mean score was $4.3 \pm 0.6$ with males scoring a bit higher (actual figures by gender not reported but $r=0.103, p<0.05$ ) [27]. Moreover, ethnicity was also a significant factor with African Americans scoring significantly higher at 4.5 (i.e. less pain) than Hispanics (4.3). Other sociodemographic variables were significantly associated with different domains of the questionnaire [27].

Other studies have also shown that culture and ethnicity have an influence on outcomes with Caucasians reporting more pain than East Asians on the SRS-30 [28, 29]. Finally, in a Polish study, the living environment has also been reported to influence the results of the SRS-24 questionnaire, with rural patients reporting more pain than those from an urban environment [30].

The Pediatric Outcomes Data Collection Instrument (PODCI), a multidimensional tool developed in North America (also known as POSNA for Pediatric Orthopedic Society of North America) [31], was used to evaluate 102 patients with AIS (as well as other groups of patients with different pathologies) [32]. Scores of these patients were compared to those of a small group of 27 "healthy" adolescents evaluated in a different study [33]. Of these $102 \mathrm{pa-}$ tients, 95 (86 girls) filled in the patient questionnaire. The scores of the two groups for the comfort/pain scale were $86.7 \pm 14.5$ for the healthy group versus $75.2 \pm 22.4$ for the AIS group. Each dimension is scaled from 0 to 100, with 100 the most favourable outcome. The result is statistically significant at $p<0.05$; however, the differences between the two groups do not seem clinically relevant when compared with the properties of the same tool described elsewhere [34].

Considering another perspective on the association between symptoms and scoliosis, a study carried out on 1743 men in the military (range: $18-30$ years) found a prevalence of idiopathic scoliosis of $6.65 \%$ among those with no symptoms and free of any lytic or olisthetic lesions [35]. Of note, none had a Cobb angle $>20^{\circ}$. Prevalence ranged from 13.3 to $23.8 \%$ in the symptomatic and asymptomatic subgroups with uni- or bilateral pars break and $18.3 \%$ among those without any lesion of the posterior arch but reporting back pain.

Clark et al. [36] have just published the results of a prospective, population-based, birth cohort study with complete data on 3184 participants. Subjects were evaluated at age 15 for scoliosis using total-body dualenergy X-ray absorptiometry (DXA) and surveyed at age 18 for pain and function. A multivariable analysis shows a significant association between small spinal curves $\left(\geq 6^{\circ}\right)$ at age 15 and self-reported back pain at age 18. Spinal curvature is also associated with days off school and avoidance of activities.

It appears quite clear from comparing the prevalence of back pain and scoliosis that the latter cannot be the main explanation for LBP for a majority of adolescents reporting such symptoms, although scoliosis playing a role in some patients cannot be ruled out. However, comparing the results of different studies does not allow any firm conclusions to be drawn on the possible causal relationship between different variables. Nevertheless, the compelling and overwhelming predominance of girls in the adolescent cohorts with idiopathic scoliosis (up to almost $90 \%$ in some series, such as that published by Théroux et al. [22]) casts doubt on the aetiological role of scoliosis on back pain in the general population, at least among boys. Another piece of information that suggests a limited role of scoliosis in back pain is the weak or even absent correlation between the magnitude of the curves measured by the Cobb angle and the presence of pain $[22,28,37,38]$.

The main references quoted in this section are summarized in Table 1.

\section{Effect of treatments on back pain}

In this section, the effects of different treatments on the magnitude of scoliotic curves have not been included. Such an analysis, as well as the impact of treatment in cosmetic, psychological or other issues, would be beyond the scope of this review. Further, long-term follow-up studies in middle-age adults have also not been included, as the role of degenerative changes cannot be identified.

\section{Conservative management}

Numerous conservative techniques have been used to treat AIS, such as acupuncture, braces, electrical stimulation, exercises, foot orthosis, osteopathy, and yoga. The most recent systematic review on braces for AIS reported long-term stability in terms of back pain (very low quality evidence) in addition to a similar outcome for quality of life and psychological and cosmetic issues [39]. The authors highlighted that performing a meta-analysis was not possible due to differences among the studies.

In a systematic review by Maruyama et al. [40], bracing was compared to observation, other conservative treatments, and surgery. Considering the effect on QoL, the evidence was considered "conflicting" for each of the 3 comparisons. As such, the authors prudently concluded only that braces "may not have a negative impact on patients' QOL". 
Table 1 Summary of the main publications including data on pain presented in the order of citation in the manuscript

N. Ramirez/JBJS 1997 [19]

Retrospective study of 2442 patients

with AIS. Mean age was 14 years for

those with back pain and 13 years for

those who did not have pain.

T. Sato/Eur Spine J 2011 [20]

Epidemiological study including 32,083 students without scoliosis and 51 with AlS. Age range was 9-15 years.

B. Lonner/Spine 2013 [21]

J. Theroux/Pain Res Manag 2015 [22]

D.W. Roberts/Spine 2011 [23]

A.J. Boniello/J Neurosurg Spine 2015 [24]

J. Bago/Eur Spine J 2009 [25]

Prospective pretreatment multicenter and retrospective chart review

including 894 patients with AIS (mean age 14.9 years) who were compared with 106 patients with Scheuermann's kyphosis (mean age 16.1 years) and with 31 healthy adolescents (mean age 14.2 years). Mean age was 13.9 years for girls and 14.5 for boys.

Longitudinal cohort study comparing outcomes before and after surgery. $N=744$ patients. Mean age was 15.2 years for boys and 14.0 in girls.

Meta-analysis of preoperative data limited to patients with Lenke type 1C curves. 1 prospective and 6 retrospective case-control studies. Overall 488 patients. Mean age for each group: 14.7 and 14.8 years (N.S.).

Study designed to identify Minimal

History of back pain

Back pain was reported by $23 \%$

at the time of presentation. Of

210 patients managed with

observation only and who were

pain-free initially, $9 \%$ reported

back pain during follow-up

(about 3 years).

Adjusted OR of back pain (point

Retrospective review of a random sample of 310 charts of AIS adolescents. important differences in 91 Als

patients undergoing surgical

procedures. Mean age was 18.1 years (range 10-38 years). or lifetime prevalence) was 2.29

with the controls. Pain was also

more severe, had longer duration and more recurrences in the scoliosis group.

Severity of back pain defined according to

(3 categories)

SRS-22

Mean Pain scores were 4.15 in the AIS group versus 4.24 among healthy controls.

Documentation of back pain from

different sources.

Previous surgery

and other spinal

pathologies were

exclusion criteria.

SRS-30

SRS-22

SRS-22

Almost half of the patients

(47.3\%) had chart-documented

pain. Pain intensity was specified

in only $21 \%$ of charts and

described as mild in $9.4 \%$

moderate in $11 \%$ and severe in

$1 \%$ of cases. Pain intensity was

Preoperatively Pain scores were

4.1 for females and 4.3 for males.

Of note, the latter were 1.2 years

older than females (average age

15.2 versus 14.0 years).

Baseline data from the largest multicenter study showed Pain scores of $4.13 \pm 0.77$ in the group eventually undergoing selective fused nonselectively.

Preop scores were 3.8-3.9 back pain, most frequently lumbar in the scoliosis group compared

Comments

Pain was associated with age

$>15$ years or Risser sign $\geq 2$ but not with gender, type or magnitude of the curves.

The difference was highly significant only for pain located in the right scapular area. No difference was found for

lumbar pain.

MCID for Pain was set at 0.2 Patients with Scheuermann kyphosis reported significantly more pain than AIS patients

No comparison group.

not correlated with the Cobb angle. fusion Vs $3.92+0.79$ in those later

Dor Pain dimensions was identified at 0.6 
Table 1 Summary of the main publications including data on pain presented in the order of citation in the manuscript (Continued)

L.Y. Carreon/Spine 2010 [26]

K. Verma/Spine 2010 [27]

L.J. Morse/Spine 2012 [28]

K. Watanabe/Spine 2007 [29]

E. Misterka/Med Sci Monit 2012 [30]

J.A. Lerman/Spine 2002 [32]

R.J. Haynes/J Pediatr Orthop 2001 [33]

L.N. Pellegrino/J Spinal Disord Tech 2014 [37]

P.R.P. Rushton/Spine 2013 [55]
Longitudinal cohort (735 girls \& 152

boys. Mean age 14.3 years) to

evaluate MCID

Healthy adolescents. Anonymous survey $N=450 / 16(10-22)$ years

Preoperative comparison of 6 ethnic groups of children with AIS. Total = 1853 composed of US white (1234), black (213), Hispanic (78), and Asian (29), as well as native Japanese (192 and Koreans (107). Overall mean age was 14.85 years ranging from 14.34 (Hispanics) to 14.97 (white). There were statistically significant

differences between groups in terms of age, gender, BMI, and major curve magnitude.

Comparison of 2 groups of 100 AIS patients each, one American and the other Japanese. Both groups were

comparable with regard to age (mean age was 15.0 years in Americans and 14.9 years in Japanese), gender, curve location, Cobb angle and thoracic

kyphosis.

Retrospective study comparing 20 rural and 40 urban Female Polish patients with AlS with $\geq 2$ years follow-up after surgery for AlS

102 adolescents with AIS (15.3 years), 47 with congenital scoliosis and 84 "normals".

Only the 1st administration of the questionnaire included. Parents' questionnaire for patients 2-18 years. Patients' questionnaire for those aged 11-18 years.

Prospective observational study preand postoperative of 33 patients (mean age 15.6 years).
SRS-22 \& SRS-30

Baseline scores for Pain: $4.1 \pm 0.71$

SRS-22

SRS-30

SRS-24

SRS-24

PODCI (Pediatric

Outcomes Data

Collection Instrument).

All dimensions scaled

0-100 where highest

is best.

POSNA (Pediatric

Orthopaedic Society

of North America)

SRS-30 and SF-36

Preop mean SRS pain score was $3.95 \pm 0.09$ and SF-36 $61.00 \pm 4.20$

Mean score for Pain domain was $4.3 \pm 0.6$. Males had higher score. African Americans scored higher than Hispanics.

The scores for the Pain domain ranged from $4.52 \pm 0.51$ among the Japanese patients, to $4.04 \pm$ 0.72 in the US white patients $(P<0.001)$

Scores for the total pain domain were $3.7 \pm 0.8$ among Americans

Mean Pain scores were 4.4 in the urban group and 4.3 in the rural one (NS).

Comfort/Pain scores self reported by adolescents $(N=95)$ (parents' questionnaires NOT included here) respectively were $86.7 \pm 14.5$ for "Normals" and 75.2+22.4 in the AIS group $(P<0.05)$
MCID for Pain domain: 0.2 and $4.3 \pm 0.4$ in the Japanese group

The authors highlight the cultural differences and suggest that a cross-cultural comparison of the SRS-24 content is necessary.

Whites reported more pain than Japanese and Koreans. The authors recommend taken into account cultural and ethnic differences when counseling patients.

Some differences between groups were found but the authors did not end-up with strong conclusions based on the environment.

No significant differences in Comfort/pain scores according o age, Cobb angle or curve location.
SRS-22r 
Table 1 Summary of the main publications including data on pain presented in the order of citation in the manuscript (Continued)

Review and statistical analysis of the

literature. Data on pain were available

from 21 cohorts from 15 published

In 17 cohorts Als patients reported

statistically more pain than controls,

in 3 cohorts patients and controls

scored similarly, and in 1 study

patients had less pain. However,

in only 1 study was the difference

clinically relevant ( $>$ MCID)

E.M. Clark/Spine 2016 [36]

Prospective, population-based, birth cohort study. Subjects with spinal

curve $\geq 6^{\circ}$ at the age $15(N=202 / 3184)$

were surveyed for back pain at age 18

ary outcome: Pain $(\geq 1$ day in the prev. month).

$2^{\text {ary }}$ outcomes: Selfreported days off

activities
At age 15202 subjects had spinal curves $\geq 6^{\circ}$. Median curve size $11^{\circ}$. Curves $\geq 25^{\circ}$ were found in 11

Spinal curves were identified participants.

Back pain was reported by $21.3 \%$ of the subjects with curves versus $16 \%$ of those without.

$\geq 7$ days off activities in the last 6 months were reported by

$21.7 \%$ of those with spinal

curves versus $12.3 \%$ of the controls. using the DXA scoliosis method. 
The authors of the most recent Cochrane review comparing surgical and conservative management of adolescent idiopathic scoliosis did not find any papers that would allow drawing conclusions regarding back pain [41]. Several other recent review articles have not specifically included information on back pain [42-46].

Osteopathic manipulative treatments in children were reviewed by Posadzki et al. [45]. Only one study of the included references concerned the treatment of scoliosis. There is currently no evidence to support such an approach for the treatment of AIS.

In his recent review paper, Kim [47] found no evidence available for yoga, Pilates, foot orthosis or acupuncture. On the contrary, he found level I and II evidence for bracing and level II evidence in favour of scoliosis specific exercises (despite some concerns such as the difficulty of attributing all the benefit to exercises due to frequent associated bracing, the order of magnitude of the correction and some doubts concerning the level of evidence of the studies) and against electrical stimulation. Indeed, only one well-designed study published in 1995 is specifically quoted in Kim's review. In their prospective controlled trial Nachemson et al. [48] compared 3 groups: 1 braced, 1 treated by electrical stimulation, 1 observed. The success rates were $74 \%$ in braced, $33 \%$ in electrical stimulation and $34 \%$ in observed. Thus the conclusion was that electrical stimulation is comparable to natural history. Kim [47] highlights the fact that the concept of correcting scoliosis by stimulating to muscles has almost disappeared but animal studies are being done to evaluate the possibilities to influence asymmetric vertebral growth by means of electrical stimulation. Only one study cited by this review addressed the impact on pain, but this study assessed exclusively non-structural scoliosis with a mean Cobb angle $<8^{\circ}[49]$.

A recent systematic review on the effects of exercise in patients with AIS concluded that exercises improve quality of life [50]. The data on pain is clearly reported in only two of the nine studies included in the review, of which both used the SR-22. In the RCT by Monticone et al. [51], at baseline, the scores in the pain dimension were $3.8(0.4)$ in the exercise group and $3.9(0.5)$ among the controls. At 1-year follow-up, the same values were $4.7(0.2)$ and $4.2(0.4)$, respectively. In the retrospective study by Noh et al. [52] (method of randomisation not clearly mentioned if any), the same scores were 4.5 (2.4) in the corrective spinal technique (combining the Schroth concepts and core stabilization techniques) and 3.8 (1.6) in the control group (conventional exercise) at the beginning of the trial and 4.9 (1) and $4.6(2.4)$, respectively, at 3 months. Notably, both groups began with 16 patients each, but at follow-up, data were reported for only 8 subjects in the experimental group and 4 patients in the control group.
A recent trial compared an 8-week program of weeklysupervised spinal stabilisation exercises to unsupervised exercises [53]. Pain, evaluated by numerical rating scale, scored 5.4 on average in each group. After 8 weeks, the improvement was 3.9 (1.8) in the supervised group versus 2.2 (2.0) in the unsupervised group. The study has clear limitations, such as the small numbers and the dropout rate in the unsupervised group, as highlighted by the authors.

The beneficial effects of bracing in terms of pain are influenced by compliance with the SOSORT Brace Treatment Management Guidelines (SBTMG) [54].

\section{Surgical treatment}

A recent review of the literature with statistical analysis included 16 cohorts with data on pain. Of these, $81 \%$ reported a statistically significant postoperative (2 years) improvement in pain, however, in only one study were the improvements clinically important [55]. Similarly, another systematic review concluded in favour of surgery to reduce the magnitude of spinal curves but stated that evidence supporting the correlation of this result with reduced pain is lacking [56].

A multicentre, prospective, consecutive clinical series reported on the prevalence and predictors of pain in AIS treated surgically [57]. Preoperative data from 1433 patients (80.4\% girls; mean curve: $56.7^{\circ}$ ) and changes in pain based on a subset of 295 cases with complete data and 2 years follow-up were reported. Pain was evaluated using the SRS-22 questionnaire. Preoperatively, $77.9 \%$ of patients reported some back pain, mainly mild to severe. The prevalence of mild to severe pain in the past 6 months improved with surgery from $78.3 \%$ of the subset $(N=295)$ preoperatively, to $68.8 \%$ at 1 year and to $64.4 \%$ at 2 years. Indeed, at follow-up, $40 \%$ of the 295 patients reported a decrease in pain, $38.6 \%$ no change and another $20.3 \%$ reported an increase in pain. Of note, while reported pain decreased with treatment, analgesic use remained unchanged. Reduction in pain and absolute pain at 2 years were both correlated with patients' perception of deformity, evaluated by means of the SAQ (Spinal Appearance Questionnaire). Such an association might be interpreted as decreasing the likelihood of a major biomechanical role of the deformity to explain pain.

The previously cited multicentre study that included 744 cases treated surgically reported outcomes at 2-years follow-up [23]. The SRS-30 pain scores improved to 4.3 in females and 4.5 among males, which was a significant difference $(p=0.003)$. Of note, although in absolute values the differences between genders were the same pre- and post-operatively (i.e. 0.2 ), the pre-operative difference was not statistically significantly [23].

Akazawa et al. [58] reported the results of a survey on health-related quality of life and LBP in a group of 80 
patients with AIS (mean age at follow-up: $47.4 \pm 6.8$ years) and a matched control group (mean age: $46.7 \pm 6.3$ years). There was no statistical difference in terms of pain $(4.3 \pm$ 0.6 in the idiopathic scoliosis group versus $4.2 \pm 0.5$ in the controls on the SRS-22 pain score) and a small difference of 1 point on the Roland \& Morris questionnaire $(2.4 \pm 4.1$ in the scoliosis group versus $1.4 \pm 3.1$ in the control group), which is below the MCID [59].

A prospective registry based study $(N=584$ patients $)$ recently reported on the prevalence of postoperative pain and its relationship with preoperative pain [60]. Patients reporting postoperative pain had significantly worse preoperative pain scores (SRS-22) with a mean of $3.8 \pm 0.8$ versus $4.2 \pm 0.7$, which is both statistically $(p<0.001)$ and clinically significant.

Another multi-centred registry study of AIS patients treated surgically ( $n=190$ with 2-year follow-up and $n=$ 77 with 5 years follow-up) reported on pain prevalence and trajectories after spinal fusion. Patients were evaluated by means of the SRS-30. Moderate to severe pain in the past month was reported by $35 \%$ of patients preoperatively. The same figure was $11 \%$ at 1-year follow-up and $15 \%$ at 2 - and 5-year follow-up. Curiously, medication usage did not change significantly: daily opioid use was reported by $1 \%$ across all time points and weekly or less frequent use of non-opioid medication also remained stable (23\% preoperatively, $25 \%$ at 2-year follow-up and $25 \%$ at 5 -year follow-up) [61].

A longitudinal cohort of 745 patients with AIS treated surgically was followed for 2 years and the results of the pre- and postoperative SRS-22 compared [62]. The pre- and postoperative pain scores were $4.16 \pm 0.71$ and $4.31 \pm 0.72$, respectively. The difference is statistically significant $(P<0.0001)$ but below the MCID. At 2 years, the correlation of the reported pain with the satisfaction score was 0.260 , slightly lower than for appearance (0.280) and for the total SRS-22 score (0.398) but higher than for activity (0.172) or mental (0.202) scores.

We have identified two further studies comparing the effects of bracing and surgery for the treatment of AIS. A short-term study using the SRS-22 questionnaire evaluated the outcome at age $16.3 \pm 1.6$ years of 109 Dutch adolescents with AIS treated either by brace, surgery, or brace followed by surgery [63]. The group treated by brace only reported significantly less pain at follow-up than the two groups that received surgery (4.5 vs 4.1 ). Moreover, there was no interaction between pain and the time passed since the end of treatment (i.e. interval at which the questionnaire was completed) for any of the groups [63]. A Danish group had reported on the outcome at 10 years of 181 patients with AIS treated either by brace or surgery [64]. Pain was assessed by means of 6 questions using VAS. The intensity of back and leg pain was mild, with values of 2.5 (0-10 scale) for the most severe back pain within the last 2 weeks for the braced patients and 2.1 in those operated. The scores of the SF-36 were compared with a Danish control cohort. The bodily pain scores were 74.6 (95 \% CI.: 68.7-80.5) in the braced subjects, and 71.4 (66.1-76.8) in the surgical cohort, which is not a significant difference. However, both values were significantly below the scores for the reference population (79.8 (77.6-82.0)) [64]. The authors of the Ste-Justine cohort did not find any major difference in self-reported pain based on preoperative characteristics, the degree of surgical correction or the distal level of fusion [65].

A recent study has shown that both the SRS-22 and SRS-24 questionnaires are able to discriminate between AIS patients with preoperative curves $>80^{\circ}$ and those $<45^{\circ}$. Specifically concerning pain, evaluated by the SRS-22 tool, the scores were $4.2 \pm 0.7$ for small curves versus $3.8 \pm 0.9$ for large $(p=0.003)$. The corresponding values obtained with the SRS-24 were $3.8 \pm 0.6$ and $3.5 \pm 0.7(p=0.002)$. Postoperatively, the same questionnaires did not show any significant difference in pain according to neither the percent correction $(<40 \% \mathrm{Vs} \geq 80 \%)$ nor when stratifying into post-operative curves $<11^{\circ}$ or $\geq 29^{\circ}$ [66].

A relationship between scoliosis and pain might be mediated by variables other than the curve itself. It has been shown that compared with asymptomatic volunteers, baseline data on lumbar stiffness evaluated by means of Lumbar Stiffness Disability Index (LSDI) correlates with pain (Pearson correlation " $r$ " LSDI versus SRS-22 Pain: -0749 ) and functional limitations (LSDI vs SRS-22 Function: $-0.760)$ in patients with spinal deformity [67].

\section{Untreated scoliosis}

The literature comparing untreated AIS with normal controls was recently reviewed and statistically analysed [68]. Twenty-one cohort studies were included that used different questionnaires (SRS-24/23/22/22r/30). Of these, $81 \%$ reported statistically worse pain than unaffected adolescents; however, in only $5 \%$ of cohorts was the difference clinically important. In comparison, in patients' self-image, almost three-quarters of the studies with a statistically significant difference were also clinically significant.

A few studies on the conservative management of adolescents with idiopathic scoliosis have included control groups not braced. In the series reported by Pham et al. [69], a subgroup of 32 patients (30 girls, age $12.5 \pm$ 1.4 years) with Cobb's angles $26.5^{\circ} \pm 2.4^{\circ}$ was left non braced until the next follow-up visit at least 3 months later and compared with patients wearing a specific brace (Chênau brace) full-time or during the night (weaning period). No differences in terms of pain were found.

Another study compared 78 patients with AIS being braced with 136 patients being observed. The two groups 
M Monticone/Eur Spine J 2014 [51]

$$
\text { Design }
$$

Conservative Treatment

S Negrini/Cochrane Database Syst Rev 2015 [39] Review including 7 studies (5 initially planned as RCTs controlled trails) with a total of 662 adolescents, other treat

T Maruyama/Physiother Theory Pract 2011 [40] Systematic review including 20 studies: 2 controlled clinical trials and 18 case-control studies. No RCTs found.

ME Alves de Araujo/J Bodyw Mov Ther 2012 [49] RCT comparing Pilatesbased therapy $(N=20)$ to weekly meetings with no therapeutic intervention $(N=11)$. Age ranged from 18 to 25 years

RCT comparing selfcorrection, task-oriented spinal exercises and education $(N=55)$ with traditional spinal exercises $(N=55)$. Evaluations prepost-treatment, and at

1 -year follow-up. Mean line at baseline 12.5 an 12.4 years respectively (NS)

DK Noh/J Back Musculoskelet Rehabil 2014 [52] Comparison of a corrective spinal technique (CST) spinal technique
approach with a conventional exercise (CE) program. Two groups of 16 AIS patients each. Mean age 13.8 years Versus 14.9 (N.S.)

KA Zapata/Pediatr Phys Ther 2015 [53]

Randomized trial comparing

Tools used for Pain

Results

Comments and 2 as prospective

PedsQL (only 1 item in one of the Back pain did not change in the 4 dimensions focus

specifically on pain)

ong term (very low quality

evidence)

Child Health Questionnaire (1

study) and VAS (1 study)

Borg CR 10

SRS-22 8 weeks of weekly supervised spinal stabilization exercises $(N=17)$ with 1-time

treatment $(N=17)$. Patients with AIS aged $10-17$ years does not influence back pain or HRQoL. Conflicting evidence reported for studies comparing in the control group (NS)

Pain scores at the 3 evaluations control group. $P<0.001$ for time, group, and interaction effects. and from 3.8 to 4.6 in CE group.

Numeric Pain Rating Scale (NPRS) Oswestry Patient-specific functional scale (PSFS) and from 5.4(1.3) to 3.4(1.7) in controls $(P \leq 0.05 \&>$ MCID)
The authors highlight that it was not possible to perform a metaanalysis due to important clinica differences among studies.

Compared with observation bracing bracing with other forms of treatment Pain decreased from 5.3(1.5) to 1.8 (1.9) $(P=0.0001)$ in the experimental group and from $4.4(2.3)$ to $3.8(2.7)$

All patients were female students with minor non-structural dorsolumbar scoliosis (Cobb angles

7.6(3.5) (Experimental) and 7.1(2.8) (Control) respectively. were: $3.8(0.4), 4.6(0.3)$, and $4.7(0.2)$ in the experimental program and $3.9(0.5), 4.3(0.3)$, and $4.2(0.4)$ in the

Pain scores improved from baseline to follow-up in both groups. Results were from 4.5 to 4.9 in CST group

The 2 groups were already small at baseline and the attrition rate was substantial with $N=8$ (CST) and 4 (CE) at follow-up. to 1.5(1.8) in the supervised group

The reduction in OSW was similar in both groups and the improvement in PSFS was higher in the supervised group but the difference between the 2 groups $<$ MCID in adults.
All the patients included in the trial had low back pain at baseline. 
Table 2 Summary of the main publications including data on the effect on pain of different treatments presented in the order of citation in the manuscript (Continued)

Surgical Management

PR Rushton/Spine 2013 [68]

MC Hawes/Disabil Rehabil 2008 [56]

Z Landman/Spine 2011 [57]

DW Roberts/Spine 2011 [23]
Cross-sectional study

followed by retrospective

case-control study to verify

the impact of a complete

rehabilitation team in

adolescent patients with

bracing ( $N=28$ AIS and

10 hyperkyphosis). Mean

age 15.8 years

Cross-sectional evaluation

of HRQoL of AIS patients

after completing treatment

Patients had been braced

$(N=36)$, treated by brace

and surgery (31), or only

by surgery (30). Mean age

16.3 years.

Review of the literature and

statistical analysis evaluating

the effect of surgery on

HROoL of adolescents with

AIS and 2 years follow-up.

Systematic review of studies on surgery for AIS with $\geq$

10 patients and followed

for $\geq 2$ years after surgery.

82 articles (5780 patients)

included.

Multicenter, prospective,

consecutive clinical series.

$N=1433$ patients.

Changes in pain assessed

in 295 patients with

complete data and 2 years

follow-up

Longitudinal multicenter cohort study to compare functional outcomes

between genders before and after surgery. $N=744$. Mean age was 15.2 years for males and 14 years for females.

SRS-22

Pain scores were $3.93+0.55$ among those treated by the team and $3.54 \pm$ 0.83 in those who were not (N.S.)

SRS-22

SRS-24

Trials' results presented as "painfree": Yes, No, or Not tested/

reported. Yes meant that most or all patients reported minimal or no pain and none reported post-op

SRS-22 (Z-test for proportions used to analyze preop and postop differences)

SRS-30 a statistically significant improvement of pain. The reduction was above MCID only in $1 / 12$ cohorts

The authors conclude that there is no evidence to support that the result in terms of reduced magnitude of the spinal curve is correlated with reduced pain.

Mild to severe pain within the last month in $73.2 \%$ preop, $53.6 \%$ at 1 year and $53.2 \%$ at 2 years. Pain at rest in $70.5 \%$ preop, $56.9 \%$ at 1 year and $60 \%$ at 2 years.

Compared with preoperative data, at 2 years $40 \%$ of patients reported a decrease in pain, $38.6 \%$ no change, and $20.3 \%$ an increase in pain.

Postop pain improved significantly in both genders from 4.1 to 4.3 in girls and from 4.3 to 4.5 in boys. The difference between genders is NS.
Other variables were significantly improved by the team management.

Pain was not significantly correlated with the time span between completing treatment and filling out the questionnaire.

$65 \%$ of articles did not include pain in their outcome. Of those who did, definitions were quite different

A significant disagreement between preoperative pain reported by physicians (44 \%) and patients (77.9\%) was found. Patients who were more satisfied with their appearance reported less pain.

The pain reduction at 2 years does not seem clinically meaningful. 
Table 2 Summary of the main publications including data on the effect on pain of different treatments presented in the order of citation in the manuscript (Continued)

LY Carreon/Spine 2011 [62]

EM Bunge/Eur Spine J $2007^{\text {a }}$ [63]

MO Andersen/Spine $2006[64]$
Case-control study to

compare healthy subjects

$(N=80)$ and non-idiopathic

$(N=56)$ scoliosis patients

and $\geq 21$ years after surgery.

Review of a prospective

multicenter database

treated surgically with

$\geq 2$ years follow-up. Age

at surgery $14.7 \pm 2$ years

registry examining

postoperative outcomes

of AIS patients.

$N=169$ at baseline, 1 and

2 years postop and $N=69$

for 5 years follow-up.

Longitudinal cohort of AIS patients treated surgically and evaluated preoperatively and 2 years postop. $N=745$. Mean age 14.2 years

Cross-sectional evaluation of HRQOL of AIS patients after completing treatment. Patients had been braced

( $N=36)$, treated by brace and surgery (31), or only

by surgery (30). Mean age 16.3 years.

Longitudinal study of consecutive AIS patients $(N=181)$ treated by brace (BT $=82$ ) or surgery

$(\mathrm{ST}=99)$. Follow-up 9.7 years
$(N=80)$ with idiopathic

registry. $N=584$ AlS patients

Prospective multicenter
Roland-Morris (RDQ)

SRS-22

Self-reported pain Vs pain free $1^{\text {st }} 6$ months post-op or 6-24 months post-op). The focus is on the patients with unexplained pain $>6$ months postoperative

SRS-30

SRS-22 and SRS satisfaction

SRS-22

\section{VAS ( 6 items)}

SF-36
Pain dimension scores were 4.3(0.6) in At long-term postoperative followAIS patients and $42(0.5)$ in controls (NS) up AIS patients had similar pain RDQ scores were 2.4(4.1) and 1.4(3.1) scores as healthy controls respectively.

The 2-year pain scores were $4.1 \pm 0.7$ in patients with postoperative pain and $4.5 \pm 0.6$ in the postop pain free group $(P<0.001)$. These 2 groups were also significantly different in terms of preoperative pain $(3.8 \pm 0.8 \mathrm{Vs} 4.1 \pm 0.7)$

Moderate to severe pain in the past month was reported preop by $35 \%$ of patients. The figures were $11 \%$ at 1 year, $15 \%$ at 2 years, and $15 \%$ at 5 -years post-surgery.

Pain often to very often at rest was reported by $43 \%$ at baseline, $5 \%$ at 1and 2 -years follow-up and $8 \%$ at final follow-up.

Mean pain domain scores improved from $4.16 \pm 0.71$ preop to $4.31 \pm 0.72$ $(P<0.0001)$

Pain and satisfaction domains were significantly correlated (Spearman 0.260)

Mean scores for Pain domain in the 3 groups were:

Brace: 4.5 (0.57)

Brace \& surg: $4.1(0.90)$

Only surgery: $4.1(0.71)$

Among the 6 VAS scores only "Do you feel leg pain right now" was significantly different with $0.5(0.2-0.9)$ in the B group and $0.2(0.0-0.3)$ in the ST group $(P=0.034)$

The results for the mean Bodily pain dimension of the SF-36 were 74.6 (BT) and 71.4 (ST) which are significantly lower than the mean of 79.8 of a 408 Danish control cohort variables evaluated (curve 
Table 2 Summary of the main publications including data on the effect on pain of different treatments presented in the order of citation in the manuscript (Continued)

Untreated

PR Rushton/Spine 2013 [55]

VM Pham/Ann Readapt Med Phys 2008 [69]

OF Ugwonali/Spine J 2004 [70]

$(N=1755)$ of the general

population.

Among the 723 patients

treated surgically, 555

completed the questionnaire.
Follow-up 10-30 years.

Controls evaluated by telephone

survey.

number of vertebrae

Review of the literature and

statistical analysis to compare

untreated adolescents with

SRS-22r

AlS with normal controls.

Comparison of 3 groups of

patients with AIS 32 without

brace, 41 treated full-time

with a corset and 35 wearing

the brace during the nigh

only. The 3 groups were

significantly different in age,

Risser, Cobb angle, etc.

Cross-sectional questionnaire- Child Health Questionnaire (CHQ based study. Patients with

AlS were braced $(N=78)$ or

observed $(N=136)$

Mean age was respectively

Parental form-28) and PODC

No differences in pain domains were found between the 2 groups despite fact that Cobb angles were significantly bigger in the braced group. Regression analysis showed a significant association of the $\mathrm{CHQ}$ bodily pain dimension and age but not with gender, cobb angle

or treatment.
No information gathered from the adolescents themselves is included in the study.

bracing does not decrease OoL
The authors conclude that

13.6 Versus 13.8 years (NS)

QoL Quality of Life, HROoL Health Related Quality of Life, PedsQI Pediatric Quality of Life Inventory, SRS Scoliosis Research Society

a this reference is included in both the Conservative management and Surgical treatment sections 
were very similar except for the magnitude of the curves (24.6 $6^{\circ}$ for the observed subjects and $34.5^{\circ}$ for those being braced, $p<0.0001)$. The authors used the parents' forms for the Child Health Questionnaire (CHQ) and the American Academy of Orthopaedic Surgeons Pediatric Outcomes Data Collection Instrument (PODCI). National normative values were available for the CHQ but not for the second instrument. No significant differences between the two groups of patients (observed vs braced) were reported for any of the pain dimensions of the two questionnaires. Moreover, when compared with the scores of healthy children, those with AIS showed similar scores on the bodily pain domain of the CHQ [70].

The main references quoted in this section are summarized in Table 2.

\section{Conclusions}

Back pain in adolescents is quite common, especially in girls. There is no doubt that some AIS patients are back pain sufferers; however, pain does not seem to be a major problem for the vast majority of adolescents with an idiopathic form of this type of deformity. Some elements from the literature suggest that the link between pain and scoliosis is not strongly linked with a biomechanical problem. In most studies, pain has not shown a strong correlation with the Cobb angle, untreated cases fare reasonably well from the perspective of back pain, there is a much greater difference between genders in the incidence of scoliosis compared with the epidemiological data on back pain, and patients' self-perception of their image correlates with pain. All these findings argue against a major aetiological role of the idiopathic scoliotic deformity of adolescents on back pain. However, the impact of pain in adults' scoliosis is entirely different $[71,72]$ and out of the scope of this review.

\section{Abbreviations \\ AIS, Adolescent idiopathic scoliosis; BP, Back pain; CHQ, Child health questionnaire; ICD, International classification of diseases; LBP, Low back pain; LSDI, Lumbar Stiffness Disability Index; MCID, Minimal clinically important difference; PODCI, Pediatric Outcomes Data Collection Instrument; POSNA, Pediatric Orthopedic Society of North America; QOL, Quality of Life; SAQ, Spinal Appearance Questionnaire; SRS, Scoliosis Research Society; VAS, Visual analogue scale}

\section{Acknowledgements}

The authors thank Ms Patricia Gongal (UK) for the linguistic support.

\section{Authors' contributions}

$\mathrm{FB}$ and $\mathrm{FB}$ decided together on the structure of the manuscript and the literature search strategies. FB produced a first draft of the review and tables that were critically read and double-checked by FP. Both authors read and approved the final manuscript.

\section{Competing interests}

The authors have no financial competing interests and the manuscript has not received any economic support. Moreover, the authors declare that they have no other competing interests (non-financial) in relation to this manuscript.

\section{Author details}

Department of Rheumatology, HFR Fribourg-Hôpital Cantonal, 1708 Fribourg, Switzerland. ${ }^{2}$ University of Geneva, Geneva, Switzerland. ${ }^{3}$ Department of Orthopedics, NYU, New York, USA. ${ }^{4}$ Spine Unit, Hospital Vall Hebron, 08035 Barcelona, Spain. ${ }^{5}$ Spine Unit Hospital Quirón, 08023 Barcelona, Spain.

Received: 4 February 2016 Accepted: 18 August 2016

Published online: 09 September 2016

\section{References}

1. Calvo-Munoz I, Gomez-Conesa A, Sanchez-Meca J. Prevalence of low back pain in children and adolescents: a meta-analysis. BMC Pediatr. 2013;13:14.

2. Dimar 2nd JR, Glassman SD, Carreon LY. Juvenile degenerative disc disease: a report of 76 cases identified by magnetic resonance imaging. Spine J. 2007;7:332-7.

3. Combs JA, Caskey PM. Back pain in children and adolescents: a retrospective review of 648 patients. South Med J. 1997;90:789-92

4. Gennari JM, Themar-Noel C, Panuel M, Bensamoun B, Deslandre C, Linglart A, et al. Adolescent spinal pain: the pediatric orthopedist's point of view. Orthop Traumatol Surg Res. 2015;101(6 Suppl):S247-50.

5. Hill $A B$. The environment and disease: association or causation? Proc R Soc Med. 1965;58:295-300.

6. Evans DW, Lucas N, Kerry R. Time, space and form: necessary for causation in health, disease and intervention? Med Health Care Philos. 2016;19:207-13.

7. Phillips CV, Goodman KJ. The missed lessons of Sir Austin Bradford Hill. Epidemiol Perspect Innov. 2004;1:3.

8. Phillips CV, Goodman KJ. Causal criteria and counterfactuals; nothing more (or less) than scientific common sense. Emerg Themes Epidemiol. 2006;3:5.

9. Schunemann H, Hill S, Guyatt G, Akl EA, Ahmed F. The GRADE approach and Bradford Hill's criteria for causation. J Epidemiol Community Health. 2011;65:392-5.

10. Fong DY, Lee CF, Cheung KM, Cheng JC, Ng BK, Lam TP, et al. A metaanalysis of the clinical effectiveness of school scoliosis screening. Spine (Phila Pa 1976). 2010;35:1061-71.

11. Konieczny MR, Senyurt H, Krauspe R. Epidemiology of adolescent idiopathic scoliosis. J Child Orthop. 2013;7:3-9.

12. Rogala EJ, Drummond DS, Scoliosis GJ. incidence and natural history. A prospective epidemiological study. J Bone Joint Surg Am. 1978;60:173-6.

13. Luk KD, Lee CF, Cheung KM, Cheng JC, Ng BK, Lam TP, et al. Clinical effectiveness of school screening for adolescent idiopathic scoliosis: a large population-based retrospective cohort study. Spine (Phila Pa 1976). 2010;35:1607-14.

14. Montgomery F, Willner S. The natural history of idiopathic scoliosis. A study of the incidence of treatment. Spine (Phila Pa 1976). 1988;13:401-4.

15. Weinstein SL. Idiopathic scoliosis. Natural history. Spine (Phila Pa 1976). 1986;11:780-3.

16. Deacon P, Berkin CR, Dickson RA. Combined idiopathic kyphosis and scoliosis. An analysis of the lateral spinal curvatures associated with Scheuermann's disease. J Bone Joint Surg (Br). 1985;67:189-92.

17. Blumenthal SL, Roach J, Herring JA. Lumbar Scheuermann's. A clinical series and classification. Spine (Phila Pa 1976). 1987;12:929-32.

18. Ochsmann EB, Escobar Pinzon CL, Letzel S, Kraus T, Michaelis M, Muenster E. Prevalence of diagnosis and direct treatment costs of back disorders in 644,773 children and youths in Germany. BMC Musculoskelet Disord. 2010;11:193.

19. Ramirez N, Johnston CE, Browne RH. The prevalence of back pain in children who have idiopathic scoliosis. J Bone Joint Surg Am. 1997;79:364-8.

20. Sato T, Hirano T, Ito T, Morita O, Kikuchi R, Endo N, et al. Back pain in adolescents with idiopathic scoliosis: epidemiological study for 43,630 pupils in Niigata City. Japan Eur Spine J. 2011;20:274-9.

21. Lonner B, Yoo A, Terran JS, Sponseller P, Samdani A, Betz R, et al. Effect of spinal deformity on adolescent quality of life: comparison of operative scheuermann kyphosis, adolescent idiopathic scoliosis, and normal controls. Spine (Phila Pa 1976). 2013;38:1049-55.

22. Theroux J, Le May S, Fortin C, Labelle H. Prevalence and management of back pain in adolescent idiopathic scoliosis patients: a retrospective study. Pain Res Manag. 2015;20:153-7.

23. Roberts DW, Savage JW, Schwartz DG, Carreon LY, Sucato DJ, Sanders JO, et al. Male-female differences in Scoliosis Research Society-30 scores in adolescent idiopathic scoliosis. Spine (Phila Pa 1976). 2011;36:E53-9. 
24. Boniello AJ, Hasan S, Yang S, Jalai CM, Worley N, Passias PG. Selective versus nonselective thoracic fusion in Lenke 1C curves: a meta-analysis of baseline characteristics and postoperative outcomes. J Neurosurg Spine. 2015;23:721-30.

25. Bago J, Perez-Grueso FJ, Les E, Hernandez P, Pellise F. Minimal important differences of the SRS-22 Patient Questionnaire following surgical treatment of idiopathic scoliosis. Eur Spine J. 2009;18:1898-904.

26. Carreon LY, Sanders JO, Diab M, Sucato DJ, Sturm PF, Glassman SD. The minimum clinically important difference in Scoliosis Research Society-22 appearance, activity, and pain domains after surgical correction of adolescent idiopathic scoliosis. Spine (Phila Pa 1976). 2010;35:2079-83.

27. Verma K, Lonner B, Hoashi JS, Lafage V, Dean L, Engel I, et al. Demographic factors affect Scoliosis Research Society-22 performance in healthy adolescents: a comparative baseline for adolescents with idiopathic scoliosis. Spine (Phila Pa 1976). 2010;35:2134-9.

28. Morse LJ, Kawakami N, Lenke LG, Sucato DJ, Sanders JO, Diab M. Culture and ethnicity influence outcomes of the Scoliosis Research Society Instrument in adolescent idiopathic scoliosis. Spine (Phila Pa 1976). 2012;37:1072-6.

29. Watanabe K, Lenke LG, Bridwell KH, Hasegawa K, Hirano T, Endo N, et al. Cross-cultural comparison of the Scoliosis Research Society outcomes instrument between American and Japanese idiopathic scoliosis patients: are there differences? Spine (Phila Pa 1976). 2007;32:2711-4.

30. Misterska E, Glowacki M, Panek S, Ignys-O'Byrne A, Glowacki J, Ignys I, et al. Effects of living environment on the postoperative Scoliosis Research Society-24 results in females with adolescent idiopathic scoliosis. Med Sci Monit. 2012;18:CR523-31.

31. Christakou A, Laiou A. Comparing the psychometric properties of the pediatric outcomes data collection instrument and the activities scales for kids: a review. J Child Health Care. 2014:18:207-14.

32. Lerman JA, Sullivan E, Haynes RJ. The Pediatric Outcomes Data Collection Instrument (PODCI) and functional assessment in patients with adolescent or juvenile idiopathic scoliosis and congenital scoliosis or kyphosis. Spine (Phila Pa 1976). 2002;27:2052-7. discussion 7-8.

33. Haynes RJ, Sullivan E. The Pediatric Orthopaedic Society of North America pediatric orthopaedic functional health questionnaire: an analysis of normals. J Pediatr Orthop. 2001;21:619-21.

34. Daltroy LH, Liang MH, Fossel AH, Goldberg MJ. The POSNA pediatric musculoskeletal functional health questionnaire: report on reliability, validity, and sensitivity to change. Pediatric Outcomes Instrument Development Group. Pediatric Orthopaedic Society of North America. J Pediatr Orthop. 1998;18:561-71.

35. Libson E, Bloom RA, Shapiro Y. Scoliosis in young men with spondylolysis or spondylolisthesis. A comparative study in symptomatic and asymptomatic subjects. Spine (Phila Pa 1976). 1984:9:445-7.

36. Clark EM, Tobias JH, Fairbank J. The impact of small spinal curves in adolescents who have not presented to secondary care: a population-based cohort study. Spine (Phila Pa 1976). 2016:41:E611-7.

37. Pellegrino LN, Avanzi O. Prospective evaluation of quality of life in adolescent idiopathic scoliosis before and after surgery. J Spinal Disord Tech. 2014;27(8):409-14.

38. Weiss HR, Goodall D. The treatment of adolescent idiopathic scoliosis (AIS) according to present evidence. A systematic review. Eur J Phys Rehabil Med. 2008;44(2):177-93.

39. Negrini S, Minozzi S, Bettany-Saltikov J, Chockalingam N, Grivas TB, Kotwicki T, et al. Braces for idiopathic scoliosis in adolescents. Cochrane Database Syst Rev. 2015;6:CD006850.

40. Maruyama T, Grivas TB, Kaspiris A. Effectiveness and outcomes of brace treatment: a systematic review. Physiother Theory Pract. 2011;27:26-42.

41. Bettany-Saltikov J, Weiss HR, Chockalingam N, Taranu R, Srinivas S, Hogg J, et al. Surgical versus non-surgical interventions in people with adolescent idiopathic scoliosis. Cochrane Database Syst Rev. 2015;4:CD010663.

42. Mordecai SC, Dabke HV. Efficacy of exercise therapy for the treatment of adolescent idiopathic scoliosis: a review of the literature. Eur Spine J. 2012:21:382-9.

43. Negrini S, De Mauroy JC, Grivas TB, Knott P, Kotwicki T, Maruyama T, et al. Actual evidence in the medical approach to adolescents with idiopathic scoliosis. Eur J Phys Rehabil Med. 2014;50:87-92.

44. Noshchenko A, Hoffecker L, Lindley EM, Burger EL, Cain CM, Patel W et al. Predictors of spine deformity progression in adolescent idiopathic scoliosis: A systematic review with meta-analysis. World J Orthop. 2015; 6:537-58.

45. Posadzki P, Lee MS, Ernst E. Osteopathic manipulative treatment for pediatric conditions: a systematic review. Pediatrics. 2013;132:140-52.
46. Romano M, Minozzi S, Zaina F, Saltikov JB, Chockalingam N, Kotwicki T, et al. Exercises for adolescent idiopathic scoliosis: a Cochrane systematic review. Spine (Phila Pa 1976). 2013;38:E883-93.

47. Kim HS. Evidence-based of nonoperative treatment in adolescent idiopathic scoliosis. Asian Spine J. 2014;8:695-702.

48. Nachemson AL, Peterson LE. Effectiveness of treatment with a brace in girls who have adolescent idiopathic scoliosis. A prospective, controlled study based on data from the Brace Study of the Scoliosis Research Society. J Bone Joint Surg Am. 1995;77:815-22.

49. Alves de Araujo ME, Bezerra da Silva E, Bragade Mello D, Cader SA, Shiguemi Inoue Salgado A, Dantas EH. The effectiveness of the Pilates method: reducing the degree of non-structural scoliosis, and improving flexibility and pain in female college students. J Bodyw Mov Ther. 2012;16:191-8.

50. Anwer S, Alghadir A, Abu Shaphe M, Anwar D. Effects of exercise on spinal deformities and quality of life in patients with adolescent idiopathic scoliosis. Biomed Res Int. 2015;2015:123848.

51. Monticone M, Ambrosini E, Cazzaniga D, Rocca B, Ferrante S. Active selfcorrection and task-oriented exercises reduce spinal deformity and improve quality of life in subjects with mild adolescent idiopathic scoliosis. Results of a randomised controlled trial. Eur Spine J. 2014;23:1204-14.

52. Noh DK, You JS, Koh JH, Kim H, Kim D, Ko SM, et al. Effects of novel corrective spinal technique on adolescent idiopathic scoliosis as assessed by radiographic imaging. J Back Musculoskelet Rehabil. 2014;27:331-8.

53. Zapata KA, Wang-Price SS, Sucato DJ, Thompson M, Trudelle-Jackson E, Lovelace-Chandler V. Spinal stabilization exercise effectiveness for low back pain in adolescent idiopathic scoliosis: a randomized trial. Pediatr Phys Ther. 2015;27:396-402.

54. Tavernaro M, Pellegrini A, Tessadri F, Zaina F, Zonta A, Negrini S. Team care to cure adolescents with braces (avoiding low quality of life, pain and bad compliance): a case-control retrospective study. 2011 SOSORT Award winner. Scoliosis. 2012;7:17

55. Rushton PR, Grevitt MP. What is the effect of surgery on the quality of life of the adolescent with adolescent idiopathic scoliosis? A review and statistical analysis of the literature. Spine (Phila Pa 1976). 2013;38:786-94.

56. Hawes MC, O'Brien JP. A century of spine surgery: what can patients expect? Disabil Rehabil. 2008:30:808-17.

57. Landman Z, Oswald T, Sanders J, Diab M. Prevalence and predictors of pain in surgical treatment of adolescent idiopathic scoliosis. Spine (Phila Pa 1976). 2011;36:825-9.

58. Akazawa T, Minami S, Kotani T, Nemoto T, Koshi T, Takahashi K. Healthrelated quality of life and low back pain of patients surgically treated for scoliosis after 21 years or more of follow-up: comparison among nonidiopathic scoliosis, idiopathic scoliosis, and healthy subjects. Spine (Phila Pa 1976). 2012;37:1899-903.

59. Maughan EF, Lewis JS. Outcome measures in chronic low back pain. Eur Spine J. 2010;19:1484-94.

60. Bastrom TP, Marks MC, Yaszay B, Newton PO. Prevalence of postoperative pain in adolescent idiopathic scoliosis and the association with preoperative pain. Spine (Phila Pa 1976). 2013;38:1848-52.

61. Sieberg CB, Simons LE, Edelstein MR, DeAngelis MR, Pielech M, Sethna N, et al. Pain prevalence and trajectories following pediatric spinal fusion surgery. J Pain. 2013;14:1694-702

62. Carreon LY, Sanders JO, Diab M, Sturm PF, Sucato DJ. Patient satisfaction after surgical correction of adolescent idiopathic scoliosis. Spine (Phila Pa 1976). 2011;36:965-8.

63. Bunge EM, Juttmann RE, de Kleuver M, van Biezen FC, de Koning HJ. Health-related quality of life in patients with adolescent idiopathic scoliosis after treatment: short-term effects after brace or surgical treatment. Eur Spine J. 2007;16:83-9.

64. Andersen MO, Christensen SB, Thomsen K Outcome at 10 years after treatment for adolescent idiopathic scoliosis. Spine (Phila Pa 1976). 2006:31:350-4.

65. Poitras B, Mayo NE, Goldberg MS, Scott S, Hanley J. The Ste-Justine adolescent idiopathic scoliosis cohort study. Part IV: surgical correction and back pain. Spine (Phila Pa 1976). 1994;19:1582-8.

66. Bastrom TP, Bartley C, Marks MC, Yaszay B, Newton PO. Post-operative perfection: ceiling effects and lack of discrimination with both SRS-22 and 24 outcomes instruments in adolescent idiopathic scoliosis patients. Spine (Phila Pa 1976). 2015:40:E1323-9.

67. Daniels AH, Smith JS, Hiratzka J, Ames CP, Bess S, Shaffrey Cl, et al. Functional limitations due to lumbar stiffness in adults with and without spinal deformity. Spine (Phila Pa 1976). 2015;40:1599-604. 
68. Rushton PR, Grevitt MP. Comparison of untreated adolescent idiopathic scoliosis with normal controls: a review and statistical analysis of the literature. Spine (Phila Pa 1976). 2013;38:778-85.

69. Pham VM, Houlliez A, Carpentier A, Herbaux B, Schill A, Thevenon A. Determination of the influence of the Cheneau brace on quality of life for adolescent with idiopathic scoliosis. Ann Readapt Med Phys. 2008;51:3-8. 9-15.

70. Ugwonali OF, Lomas G, Choe JC, Hyman JE, Lee FY, Vitale MG, et al. Effect of bracing on the quality of life of adolescents with idiopathic scoliosis. Spine J. 2004;4:254-60.

71. Bess S, Line B, Fu KM, McCarthy I, Lafage V, Schwab F, et al. The health impact of symptomatic adult spinal deformity: comparison of deformity types to United States population norms and chronic diseases. Spine (Phila Pa 1976). 2016;41:224-33.

72. Pellise F, Vila-Casademunt A, Ferrer M, Domingo-Sabat M, Bago J, Perez-Grueso FJ, et al. Impact on health related quality of life of adult spinal deformity (ASD) compared with other chronic conditions. Eur Spine J. 2015;24:3-11.

Submit your next manuscript to BioMed Central and we will help you at every step:

- We accept pre-submission inquiries

- Our selector tool helps you to find the most relevant journal

- We provide round the clock customer support

- Convenient online submission

- Thorough peer review

- Inclusion in PubMed and all major indexing services

- Maximum visibility for your research

Submit your manuscript at www.biomedcentral.com/submit
Biomed Central 